\section{REVISTA BRASILEIRA DE QUALIDADE DE VIDA}

\title{
Dançaterapia e a qualidade de vida de pessoas com deficiência física: ensaio clínico controlado
}

\section{Dance therapy and quality of life in individuals with physical deficit: randomized controlled trial}

\author{
Lavinia Teixeira-Machado \\ Universidade Federal de Sergipe - UFS - Aracaju - Brasil \\ laviniateixeira@infonet.com.br \\ Josimari DeSantana \\ Universidade Federal de Sergipe - UFS - Aracaju - Brasil \\ josimelo@infonet.com.br
}

\section{RESUMO}

OBJETIVO: Analisar o efeito da dançaterapia na qualidade de vida de indivíduos com deficiência física.

MÉTODOS: Este ensaio clínico, controlado e com distribuição aleatória foi composto por dois grupos: Dançaterapia e Cinesioterapia. Ambos os grupos foram tratados com duração de uma hora, duas vezes por semana, por um período de dois meses. O instrumento de avaliação utilizado foi o Questionário de Qualidade de Vida SF-36. Os procedimentos aplicados nos grupos Dançaterapia e Cinesioterapia foram realizados em locais adequados para as respectivas condutas.

RESULTADOS: Vinte e seis indivíduos foram proporcionalmente distribuídos nos dois grupos de pesquisa. Os resultados encontrados apontam comprometimento na qualidade de vida nos diversos domínios em ambos os grupos quando analisados através do SF-36. Os piores escores de qualidade de vida foram observados quanto ao domínio capacidade funcional em ambos os grupos (Dançaterapia: 20,76 \pm 6,76; Cinesioterapia: 37,69 $\pm 8,05)$. Observou-se diferença estatisticamente significativa para os itens capacidade funcional $(\mathrm{p}=0,019)$, estado geral de saúde $(\mathrm{p}=0,015)$, aspectos sociais $(\mathrm{p}=0,002)$ e limitações por aspectos emocionais $(\mathrm{p}=0,039)$ apenas para o grupo Dançaterapia.

CONCLUSÕES: A dança, como terapia, promoveu melhora na qualidade de vida de pacientes com transtornos neuromotores de origem central.

PALAVRAS-CHAVE: Terapia através da dança. Reabilitação. Encefalopatias. Hipertonia muscular. Qualidade de Vida.

\section{ABSTRACT}

OBJECTIVE: To analyze the effect of Dance therapy on quality of life in individuals with neuromotor disorders. 
METHODS: This controlled and randomized clinical trial was composed by two groups: Dancetherapy and Kinesiotherapy. Both of them were undergone to a 1-hour treatment, twice a week, along two months. The Short-Form 36 questionnaire about Quality of Life (Brazilian version) was used as an instrument of evaluation. The applied procedures in the groups Dancetherapy and Kinesiotherapy were conducted in suitable locations for the therapies.

RESULTS: Twenty-six subjects were proportionally allocated into those two groups of study. The findings suggest impairment in quality of life in various rules in both groups when analyzed through SF-36. The worst scores of this criterion were observed into the physical limitation field in both groups (Dancetherapy 20.76 \pm 6.76 , Kinesiotherapy 37.69 \pm 8.05 ). A significant difference was observed through the following items: functional capacity $(\mathrm{p}=.019)$; general health $(\mathrm{p}=.015)$; social aspects $(\mathrm{p}=.002)$ and limitation for emotional aspects $(\mathrm{p}=.039)$ only for the group Dancetherapy after treatment.

CONCLUSIONS: This group promoted enhancement on the quality of life in patients with brainrelated neuromotor disabilities.

KEYWORDS: Dance therapy. Rehabilitation. Brain diseases. Muscle hypertonia. Quality of life.

\section{Introdução}

O indivíduo age no mundo através do seu corpo (PINHEIRO FILHO, 2004). O movimento possibilita que as pessoas se comuniquem, trabalhem, aprendam, sintam o mundo e sejam sentidas (KATZ, 2005; STRAZZACAPPA, 2001).

Indivíduos com limitações motoras, causadas por doenças neurológicas, apresentam dificuldades de evidenciar e exprimir sua individualidade perante a sociedade (RESENDE, 2008). Uma forma de se potencializar a percepção e o processamento do meio externo é mediante estímulos teleceptivos, ou seja, ativação dos sentidos visuais e auditivos e do sistema vestibular (CARR; SHEPHERD, 2011; MOHAPATRA; ARUIN, 2013), os quais ativam regiões cerebrais responsáveis por enviar informações das vias sensoriais para as vias motoras (DAPRATI; SIRIGU; NICO, 2010).

Mediante esses estímulos, busca-se o eixo da intervenção pela dançaterapia. A música sugere que os movimentos acompanhem seus tons melódicos, os quais imprimem contextos emocionais que são transmitidos para a plateia atenta. Assim, as vias sensoriais são potencializadas e, como ressalta Duignan, Hedley e Milverton (2009), sua ação não é só psicomotora, mas também psicossocial.

Ademais, a dança proporciona saúde e bem-estar biológico, psicológico e social (SEIXAS, 2005); a dança, como terapia, enquadra-se nos preceitos de saúde pública, por promover saúde, prevenir doenças e proporcionar a longevidade (DUIGNAN; HEDLEY; MILVERTON, 2009).

O conceito de saúde e qualidade de vida (QV) em pessoas com deficiência é mais complexo. O interesse acerca da inclusão desses indivíduos no cenário social envolve uma série de contextos estruturados pelas diferenças e pelos antagonismos, como caracteriza Hall (2001). A inserção nos contextos sociais do indivíduo com limitações físicas anula o sentido discriminativo ou estigmático, e permite a autoestima a partir do espaço social que lhe é conferido ou obtido (VELHO, 2008).

As doenças neurológicas reduzem a QV (HACKNEY; EARHART, 2009), acometendo os aspectos físico, cognitivo, emocional e social (HACKNEY; EARHART, 2009; SCHIMITT; FRÖLICH, 2007). Lesões cerebrais debilitam orientação espaço-temporal e cognitiva, e isso se reflete na dificuldade de interagir e de posicionar-se perante uma situação, o que repercute nas atividades do cotidiano e consequentemente em QV satisfatória (SCHIMITT; FRÖLICH, 2007).

Novos paradigmas vêm influenciando as ações no âmbito da saúde. A preocupação com o conceito de QV configura as estratégias que embasam os parâmetros promotores do estado de bemestar do ser humano, modificando as concepções referentes ao processo de saúde e doença, mediante a experiência pessoal e o estilo de vida de cada indivíduo em particular. Para tal, a 
melhoria da QV tornou-se foco de interesse das práticas assistenciais das políticas públicas para promoção e prevenção de agravos à saúde, relacionada aos aspectos econômicos e socioculturais (SCHUTTINGA, 1995).

Nas últimas décadas, a QV vem sendo incluída como um indicador de saúde para avaliar a qualidade dos serviços no âmbito da saúde coletiva e das políticas públicas, além da eficácia, eficiência e o impacto causado por determinadas estratégias. Assim, as informações extraídas dos instrumentos que avaliam a QV visam minimizar problemas e preencher lacunas em procedimentos que não obtiveram sucesso (KAPLAN, 1995).

Os achados referentes à QV também auxiliam as práticas assistenciais cotidianas dos serviços de saúde, no sentido de propor julgamentos clínicos nas especificidades das condições de saúde. Isto porque a QV permite melhor visualização das enfermidades, disfunções e incapacidades que causam impacto físico e psicossocial, além de influenciar as condutas propostas pelas equipes de saúde (MORRIS; PEREZ; NOE, 1998).

Condutas terapêuticas que fomentem a inserção de pessoas com deficiência no contexto sociocultural são de fundamental importância (SEIXAS, 2005). A terapia pela dança permite exploração do espaço, transforma o ambiente e gera autoconfiança, autonomia e independência (CAZE; OLIVEIRA, 2008).

Isso porque a dança impulsiona a sensibilidade inerente ao homem e inquire a corporeidade. Expondo de maneira superficial, corporeidade é a capacidade do corpo pensar, pensamento este que conduz um movimento para representá-lo. E isso se reflete num movimento organizado (FORINASH, 2005; KATZ, 2005; NAONE, 2007; RESENDE, 2008; ROUHIAINEN, 2007; XIA; GRANT, 2009).

Propor tarefas que fomentem o movimento como veículo primário para garantir destreza inata é diretamente aplicável em desordens restritivas (SAPER, 2002). Oferecer melhora do repertório do movimento humano, assim como redução da dor e limitação articular, viabiliza a expansão das escolhas pessoais e respostas para vários aspectos da vida - emocionais, relacionais e intelectuais (BUCHANAN; ULRICH, 2001) —, por contribuir para um estado de bem-estar em vários níveis da percepção humana (DAPRATI; SIRIGU; NICO, 2010).

Ao aprender ou reaprender a realizar uma função motora, uma pessoa experimenta, nas áreas sensoriais somáticas, os efeitos do movimento cada vez que é realizado, e experiências dos diferentes padrões de ação são registradas (LABAN, 1984; SAPER, 2002; STEPHENS; MILLER, 2009). Atividades como a dança envolvem a música e a percepção visual do corpo (pelo espelho) para que este se movimente de acordo com as solicitações advindas da subjetividade e para que a ação motora ocorra no tempo-espaço pré-determinado (CARR; SHEPHERD, 2011; FRONTERA; SILVER, 2002; LLINÁS, 2002; DAPRATI; SIRIGU; NICO, 2010; RIMMER, 2008).

A dança, além de evidenciar ações que se assemelham a movimentos empregados no esporte, em atividades de trabalho e da vida diária, fomenta um dos princípios da aprendizagem psicomotora: o equilíbrio estático e dinâmico corpóreo, de fundamental importância para a orientação espaço-temporal, bem como a percepção do posicionamento ou de um segmento do corpo no espaço (TEIXEIRA-MACHADO, 2011).

Atividades rítmicas, como a dança, propõem estruturação e organização em tempo, espaço, ordem e dinâmica (STEPHENS; MILLER, 2009; TAUBE, 1998). A terapia pelo movimento recupera ou desperta memórias pré-verbais no corpo, através de sensações corporais que permitem a construção ou reconstrução mediante ação mnemônica pré-verbal e não-verbal (FUJITA, 2005).

A dança abrange atividades expressivas, sensitivas, sensoriais, criativas, motoras e rítmicas e, assim, propõe o conhecimento do próprio corpo e de suas potencialidades quanto à percepção e à expressão do que é percebido e sentido (XIA; GRANT, 2009).

Desta forma, oferecer novas propostas de procedimentos terapêuticos que visam o bem-estar de pessoas com deficiência física, aliados aos benefícios alcançados, mediante a prática da dança, que proporcionam um aparato neuromotor mais funcional, foram o enfoque deste estudo, assim como propor novas alternativas terapêuticas que gerem motivação e, consequentemente, melhora da QV. 
A escassez de estudos nesta área instigou a necessidade de vislumbrar possíveis contribuições que a dança como terapia possa gerar em indivíduos com limitações neuromusculares. Assim, o estudo objetivou analisar os efeitos da dança terapêutica na QV de pessoas com deficiência física.

\section{Métodos}

A pesquisa está plenamente adequada às Diretrizes e Normas de Pesquisa em Seres Humanos do Conselho Nacional de Saúde (item VII da Resolução 196/96), pelo Comitê Nacional de Ética e Pesquisa (CONEP) e aprovada pelo Comitê de Ética e Pesquisa da Universidade Federal de Sergipe (CEP/UFS), no 0135.0.107.000-09. O Termo de Consentimento Livre e Esclarecido foi devidamente assinado por todos os sujeitos envolvidos no estudo.

\subsection{Delineamento do Estudo}

Esse estudo é um ensaio clínico, controlado e com distribuição aleatória. Uma distribuição bloqueada foi realizada para gerar uma sequência de alocação para garantir proporcionalidade de distribuição entre dois grupos durante todo o desenvolvimento do estudo. Assim, o número de participantes em cada grupo seguiu a proporção 1:1.

As pessoas com deficiência física foram aleatoriamente incluídas em um dos dois grupos de estudo. Para tal, a distribuição aleatória ocorreu na ordem em que os sujeitos foram incluídos no estudo, de acordo com uma distribuição gerada por computador, a qual foi preparada antes de iniciar a coleta de dados.

\subsection{Casuística}

Foram considerados como critérios de inclusão para esta amostra: pessoas com deficiência motora por lesão nervosa cerebral; ambos os gêneros; faixa etária entre 15 e 29 anos; presença de hipertonia muscular determinada ao teste de movimentação passiva; que não realizasse, paralelamente ao desenvolvimento do protocolo de pesquisa, outra atividade física; que não apresentasse cardiopatias ou neoplasias. Alterações cognitivas ou psiquiátricas associadas e a recusa em permanecer participando do estudo constituíram critérios de exclusão.

Os sujeitos foram aleatoriamente alocados em um dos seguintes grupos: Grupo Dançaterapia, composto por sujeitos com deficiência física, submetidos à dançaterapia; e Grupo Cinesioterapia, formado por pessoas que possuíam deficiência física submetidas a atendimento cinesioterapêutico conforme técnicas tradicionais, caracterizando, assim, o grupo controle.

Uma estimativa do tamanho amostral pretendido para os grupos de estudo foi determinada usando os dados dos primeiros 12 sujeitos envolvidos no estudo. Um $\alpha=0,05$ e um poder $=75 \%$ indicou um tamanho amostral de 12 sujeitos por grupo.

\subsection{Protocolos de estudo}

Os Grupos Dançaterapia e Cinesioterapia receberam os mesmos procedimentos, com ênfase nos conceitos de Feldenkrais (BUCHANAN; ULRICH, 2001; MEHLING et al., 2011), Bobath (KIM et al., 2012) e facilitação neuromuscular proprioceptiva (EL-TAMAWY; DARWISH; KHALLAF, 2012) com duração de uma hora, duas vezes por semana, num período de dois meses. As sessões foram divididas em quatro módulos: aquecimento, treino de flexibilidade, treino de equilíbrio, relaxamento. Apenas no Grupo Dançaterapia utilizava-se a música como auxiliar nas tarefas realizadas, como também apresentações públicas dançadas em escolas, unidades de saúde, praças, teatros. 


\subsection{Instrumentos de avaliação}

Uma ficha de avaliação foi utilizada no início do estudo para a coleta de dados demográficos (nome, idade, peso, ocupação profissional, estado civil, cor, história da doença atual, história da doença pregressa, antecedentes familiares, história fisiológica, história social) e registro de informações relacionadas às atividades funcionais dos sujeitos da pesquisa.

Para a avaliação da QV foi utilizada a versão brasileira do Questionário sobre QV Medical Outcomes Study 36 - Item Short Form Health Survey (SF-36). A versão brasileira do SF-36 é muito utilizada em várias áreas da saúde para análise da QV (CICONELLI et al., 1999).

O questionário SF-36 inclui uma escala multi-item que acessa oito dimensões:

a) limitações na atividade física, devido a problemas de saúde;

b) limitações na atividade social, devido a problemas físicos e emocionais;

c) limitações nas atividades usuais, devido a problemas físicos de saúde;

d) dor corporal;

e) saúde mental geral (estresse psicológico e bem-estar);

f) limitações nas atividades, devido a problemas emocionais;

g) vitalidade (energia e fadiga);

h) percepção da saúde geral.

As questões são designadas para um fácil entendimento e relevante para a maioria das pessoas. São requeridos cerca de cinco a dez minutos para completar os questionamentos. O Questionário SF-36 (versão brasileira) foi aplicado em dois momentos: antes de iniciar as sessões de Dançaterapia e de Cinesioterapia e após dois meses de realização do protocolo.

\subsection{Execução do protocolo}

O procedimento aplicado no grupo Dançaterapia foi realizado em sala contendo barras fixadas à parede, espelho e equipamento de som, onde foram executadas aulas de dança (atividade rítmica coordenada), com duração de uma hora, duas vezes por semana, durante dois meses. A conduta aplicada no grupo Cinesioterapia foi realizada em ambulatório devidamente preparado para o atendimento da cinesioterapia, no Centro de Reabilitação Ninota Garcia da Universidade Tiradentes, Sergipe (Figura 1).

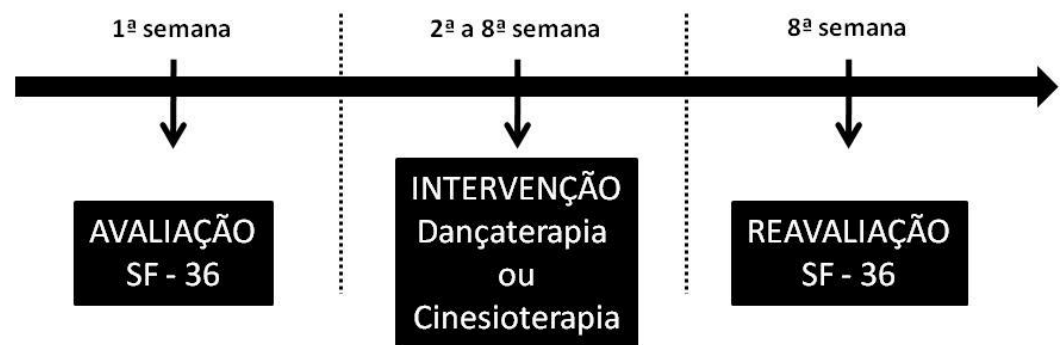

Figura 1 - Linha do tempo do protocolo de pesquisa Fonte: Autoria própria (2013).

\subsection{Análise estatística}

Os dados coletados foram inicialmente transportados para uma planilha de dados do programa Excel for Windows 2007 e, então, para o programa SPSS, versão 16.0, para as seguintes análises:

a) descritiva: com confecção de tabelas de frequência, medidas de posição (média, mínima, máxima) e dispersão (erro-padrão);

b) de comparação: teste T-Student para amostras dependentes e independentes. Os dados com valor de $\mathrm{p} \leq 0,05$ foram considerados estatisticamente significativos. 


\section{Resultados}

Trinta e dois sujeitos foram elegíveis para iniciarem participação no estudo. Dois sujeitos foram excluídos da pesquisa por apresentarem idade inferior a treze anos. Assim, 30 indivíduos iniciaram a pesquisa. Durante a execução dos protocolos, dois indivíduos concluíram as sessões de cinesioterapia e dois desistiram de participar da pesquisa. Vinte e seis indivíduos foram distribuídos nos dois grupos de pesquisa. Treze sujeitos foram alocados no grupo Cinesioterapia, cinco do gênero feminino e oito do gênero masculino. Treze sujeitos foram incluídos no grupo Dançaterapia, sendo sete do gênero feminino e seis do gênero masculino (Figura 2).

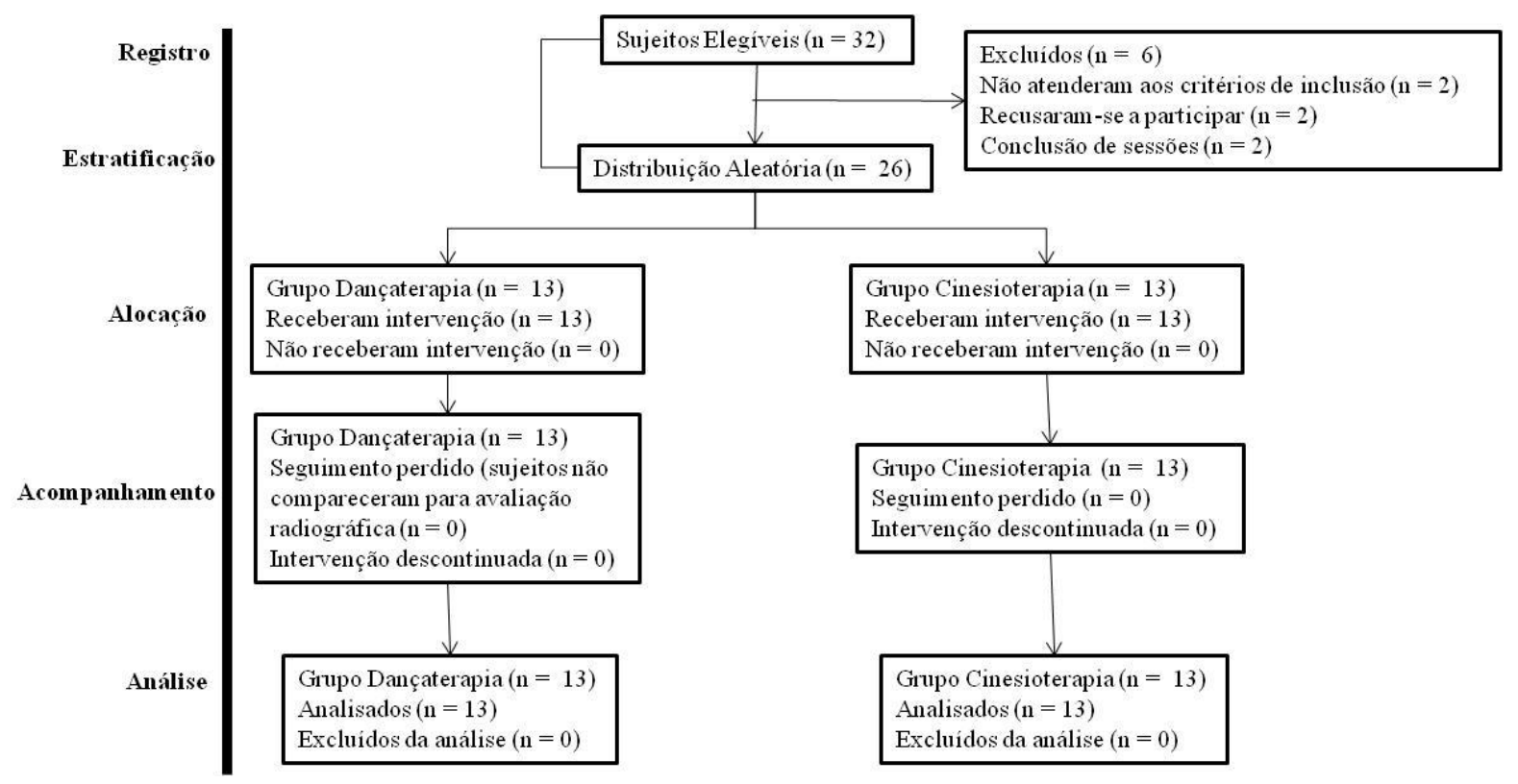

Figura 2 - Elegibilidade e distribuição dos sujeitos nos grupos de pesquisa Fonte: Autoria própria (2013).

Os dados referentes a idade e peso dos sujeitos foram estatisticamente semelhantes entre os grupos (Tabela 1).

Tabela 1 - Idade (anos) e peso $(\mathrm{Kg})$ dos sujeitos incluídos nos Grupos Dançaterapia e Cinesioterapia

\begin{tabular}{cccc}
\hline Característica Demográfica & Dançaterapia & Cinesioterapia & P \\
\hline Idade (anos) & $20,46 \pm 2,11$ & $20,61 \pm 3,21$ & 0,88 \\
Peso $(\mathrm{Kg})$ & $58,77 \pm 6,53$ & $59,54 \pm 7,22$ & 0,79 \\
\hline
\end{tabular}

Nota: Média \pm erro padrão da média e valores de P. Teste-t Student para amostras Independentes

Fonte: Autoria própria (2013).

Os resultados encontrados apontam comprometimento na QV nos diversos domínios, em ambos os grupos, quando analisados através do SF-36. Os piores escores de QV foram observados no domínio capacidade funcional em ambos os grupos (Dançaterapia: 20,76 $\pm 6,76$; Cinesioterapia: $37,69 \pm 8,05$; Tabelas 1 e 2). 
Tabela 2 - Domínios do Questionário SF - 36 antes e depois da intervenção com Cinesioterapia

\begin{tabular}{llll}
\hline \multicolumn{1}{c}{ Domínios do SF - 36 } & Etapas da Intervenção com Cinesioterapia & P \\
\hline & ANTES & DEPOIS & \\
Capacidade funcional & $37,69 \pm 8,05$ & $39,23 \pm 7,67$ & 0,16 \\
Limitação por aspectos físicos & $46,15 \pm 8,41$ & $46,15 \pm 8,41$ & 1,00 \\
Dor & $74,92 \pm 6,08$ & $75,69 \pm 6,60$ & 0,34 \\
Estado geral da saúde & $56,07 \pm 1,41$ & $56,07 \pm 1,41$ & 1,00 \\
Vitalidade & $60,38 \pm 3,60$ & $61,53 \pm 3,50$ & 0,19 \\
Aspectos Sociais & $59,61 \pm 7,23$ & $59,61 \pm 7,23$ & 1,00 \\
Limitações por aspectos emocionais & $51,00 \pm 10,40$ & $51,00 \pm 10,40$ & 1,00 \\
Saúde mental & $79,69 \pm 2,14$ & $79,69 \pm 2,14$ & 1,00 \\
\hline
\end{tabular}

Nota: Média \pm erro padrão da média e valor de P. Teste t para amostras dependentes.

Fonte: Autoria própria (2013).

Tabela 3 - Domínios do Questionário SF - 36 antes e depois da intervenção com Dançaterapia

\begin{tabular}{lccc}
\hline \multicolumn{1}{c}{ Domínios do SF - 36 } & Etapas da Intervenção com Dançaterapia & P \\
\hline & ANTES & DEPOIS & \\
Capacidade funcional & $20,76 \pm 6,76$ & $30,76 \pm 6,83$ & $0,02 *$ \\
Limitação por aspectos físicos & $46,15 \pm 10,14$ & $53,84 \pm 10,53$ & 0,22 \\
Dor & $55,23 \pm 9,42$ & $57,00 \pm 9,69$ & 0,66 \\
Estado geral da saúde & $50,76 \pm 3,38$ & $57,53 \pm 2,45$ & $0,01 \dagger$ \\
Vitalidade & $41,92 \pm 5,64$ & $41,53 \pm 5,50$ & 0,86 \\
Aspectos Sociais & $60,57 \pm 9,15$ & $72,11 \pm 9,82$ & $0,01 *$ \\
Limitações por aspectos emocionais & $63,69 \pm 8,81$ & $74,15 \pm 10,1$ & $0,03 *$ \\
Saúde mental & $45,84 \pm 2,29$ & $49,84 \pm 3,05$ & 0,13 \\
\hline Nota: Média erro padrão da média e valor de P. Teste t para amostras dependentes, $*$ p< 0,$05 ;$ \\
†p $\leq 0,01$. \\
Fonte: Autoria própria (2013).
\end{tabular}

A partir da análise dos domínios do Questionário SF-36, observou-se diferença estatisticamente significativa para os itens capacidade funcional $(\mathrm{p}=0,019)$, estado geral de saúde $(\mathrm{p}=0,015)$, aspectos sociais $(\mathrm{p}=0,002)$ e limitações por aspectos emocionais $(\mathrm{p}=0,039)$ no grupo Dançaterapia. Houve melhora na capacidade funcional, na vitalidade e redução da dor para o grupo Cinesioterapia, embora estes resultados não tenham apresentado significância estatística (Tabelas 2 e 3).

\section{Discussão}

Os resultados desse estudo evidenciaram comprometimento nas diferentes dimensões analisadas através do Questionário SF-36, sendo que os menores valores médios foram observados na dimensão capacidade funcional.

É importante frisar que essa dimensão avalia, principalmente, o desempenho nas atividades físicas motoras diárias e de trabalho. A redução desse desempenho compõe sintomas frequentes em pacientes com sequelas de lesão cerebral.

Esses achados são semelhantes aos descritos por Duignan, Hedley e Milverton (2009), que evidenciam a redução da ansiedade, sem mudanças significativas na capacidade funcional, e por Hackney e Earhart (2009) no auxílio da dançaterapia ao equilíbrio corporal.

A maioria dos estudos com desenho metodológico similar aborda os benefícios da dançaterapia em idosos (ALPERT et al., 2009; D'ALENCAR et al., 2008; JEON et al., 2005; NYSTRÖM; LAURITZEN, 2005), adolescentes com transtornos alimentares (LAUMER et al., 1997), no manejo do câncer (AKTAS; OGCE, 2005; COHEN; WALCO, 1999; SANDEL et al., 2005), na deficiência cognitiva e mental (DUIGNAN; HEDLEY; MILVERTON, 2009; NYSTRÖM; LAURITZEN, 2005; PALO-BENGTSSON; WINBLAD; EKMAN, 1998; SCHMITT; FRÖLICH, 2007), na depressão (ALPERT et al., 2009; JEONG et al., 2005; TSANG; CHAN; CHEUNG, 2008), em surdos (PELC, 2002) e em transtornos de equilíbrio, principalmente os 
resultantes de doenças como Parkinson e Alzheimer (HACKNEY et al., 2007; HACKNEY; EARHART, 2009; RÖSLER et al., 2002).

Os estudos que abordaram a QV nos distúrbios neurológicos, compararam os efeitos da dançaterapia com outras atividades físicas. Os instrumentos de avaliação da QV foram questionários estruturados e semi-estruturados direcionados à doença associada. Não foi encontrado um protocolo de avaliação da QV para pessoas com deficiência física de origem central, apenas um protocolo específico para indivíduos com doença de Parkinson (D'ALENCAR et al., 2008; DUIGNAN; HEDLEY; MILVERTON, 2009; HACKNEY; EARHART, 2009). Nenhum dos autores utilizou o questionário SF-36.

No Grupo Dançaterapia houve uma melhora significativa nos domínios capacidade funcional, estado geral de saúde, aspectos sociais e limitações por aspectos emocionais.

Em relação aos dados encontrados, referentes aos aspectos emocionais, Xia e Grant (2009) afirmam que a dança abrange atividades expressivas, sensitivas, sensoriais, criativas, motoras e rítmicas, e, assim, propõe o conhecimento do próprio corpo e de suas potencialidades quanto à percepção e expressão do que se é percebido e sentido.

As atividades propostas pela cinesioterpia, apesar de efetivas, não ativam os estados emocionais, como percebido no grupo dançaterapia. Segundo Forinash (2005), Loman (2005) e Pang et al. (2006), o ritmo acompanhado de movimento fomenta percepção analítica e simbólica e, portanto, permite localização e estruturação da dimensão temporal, assim como a percepção, a criação e a expressão de um movimento desenvolvido num espaço-tempo próprio e natural.

Quando Naone (2007), Resende (2008) e Rouhiainen (2007) afirmam que o estado estético impulsiona a sensibilidade inerente ao homem e inquire a corporeidade, a capacidade funcional é vislumbrada pela dança, pois considera a complexidade e a singularidade anatômica e cinética de cada um.

A melhora significativa da capacidade funcional no Grupo Dançaterapia revela que a terapia pela dança permite descobrir novos caminhos para melhor utilização do corpo (NAONE, 2007), não só pelo fato de usar componentes importantes para ação motriz, como equilíbrio, postura, coordenação, destreza (LOMAN, 2005), mas, também, por desenvolver atividades de vida diária.

Do ponto de vista estatístico, os resultados encontrados não apresentaram significância no Grupo Cinesioterapia. Já no Grupo Dançaterapia, não houve significância nos itens limitação por aspectos físicos, intensidade da dor, vitalidade e saúde mental. Entretanto, do ponto de vista clínico, alguns parâmetros do questionário não são apropriados para analisar possíveis mudanças ocorridas no decorrer das sessões que os sujeitos foram submetidos em ambos os grupos.

Por exemplo, o item limitação por aspectos físicos questiona sobre atividades como correr, participar de esportes, levantar ou carregar mantimentos, mover mesa, subir escadas, que não são executadas, na maioria das vezes, por pessoas com injúrias do sistema nervoso central (MOHAPATRA; KRISHNAN; ARUIN, 2012), como percebido na amostra do presente estudo.

As sequelas por lesão central dificultam e, em alguns casos, incapacitam a manipulação de objetos e comprometem o organismo como um todo (CARR; SHEPHERD, 2012; FRONTERA; SILVER, 2002; CICONELLI et al., 1999; DAPRATI; SIRIGU; NICO, 2010). A maioria dos sujeitos da pesquisa não deambula independentemente, outros tiveram lesões em regiões responsáveis pelo controle do movimento, as quais comprometem a destreza e a organização de padrões motores normais.

Considerando os achados referentes aos sujeitos que receberam tratamento baseado em dança, pode-se sugerir que a dança potencializa a percepção e o processamento do meio externo, por utilizar veementemente estímulos teleceptivos (visuais, auditivos, vestibulares).

Segundo Kandel (2001) e Llinás (2002), esses estímulos ativam regiões cerebrais que transmitem informações pelas vias sensoriais. Devido ao seu poder narrativo e mnemônico (LOMAN, 2005; SACKS, 2006), atividades rítmicas coordenadas permitem a exploração do espaço, transforma o ambiente e gera autoconfiança, autonomia e independência (KATZ, 2005; NAONE, 2007; STEPHENS; MILLER, 2009). 
O estado geral da saúde engloba a percepção de saúde do questionado. Os itens que compõem essa dimensão abordam perguntas acerca da concepção que o participante tem em relação a situações e agravos à saúde que possam vir a acontecer, ou seja, abrange o sentido de otimismo ou pessimismo referente à saúde do questionado.

Assim, os aspectos emocionais evidenciados no universo da afetividade que a dança propõe, principalmente motivação e prazer em se expressar corporalmente, salientou o estímulo em interagir e demonstrar as capacidades emotivas pela dança. E isso era observado claramente no momento em que esses 'deficientes', estigmatizados pela sociedade como um todo, inclusive por eles próprios, como 'incapazes', provocavam lágrimas que evocavam sentimentos de auto-avaliação da plateia 'sem deficiências'.

Essas experiências particulares embutem um valor simbólico tão devastador na subjetividade da pessoa com deficiência que não há como mensurar nem quantificar os efeitos na alteridade. A aceitação e a exaltação da diferença estimula a autonomia e a autoconfiança. Ademais, Schimitt e Frölich (2007) elucidaram a integração dos processos sensoriomotores mediante a intervenção pela dançaterapia. Alguns estudos indicaram os benefícios da dança em relação à depressão em pacientes oncológicos (AKTAS; OGCE, 2005), idosos (JEON et al., 2005) e adolescentes depressivos (JEONG et al., 2005).

É uma necessidade humana ser percebido pelo outro, ser aplaudido por algo que realizou. E a dança contribui para isso, pois evidencia aquele que está em um palco ou numa situação de destaque, em que todos à sua volta estão observando-o e o avaliando.

A depressão geralmente está associada a alterações comportamentais de fuga às questões que norteiam o indivíduo de ser valorizado no meio em que vive (PELC, 2002). Quando a integração com o mundo exterior provoca sinais de incapacidade, o ser se retrai e inicia-se um processo de isolamento por 'achar' que suas particularidades não são aceitas no contexto biopsicossocial.

A integração do indivíduo com o mundo exterior proposta pela dança influencia o processo de socialização, como extensão da capacidade funcional de ser parte de um todo, principalmente no contexto social que interfere no cenário comportamental da pessoa com deficiência (D'ALENCAR et al., 2008; PELC, 2002).

A dança como terapia contribui para a inserção do indivíduo com limitações físicas nos contextos sociais, como proposto por Velho (2008). Essas afirmações concatenam as ideias de Buchanan e Ulrich (2001) e Daprati, Sirigu e Nico (2010) acerca da expansão das escolhas pessoais e respostas para vários aspectos da vida (sejam eles emocionais, relacionais ou intelectuais) e determinam os resultados satisfatórios por permitir estado de bem-estar em vários níveis da percepção humana, principalmente num corpo com dificuldades e limitações de expressão.

Embora diversos autores tenham relatado melhora significativa nos aspectos emocionais, funcionais e sociais da QV (AKTAS; OGCE, 2005; ALPERT et al., 2009; D'ALENCAR et al., 2008; DUIGNAN; HEDLEY; MILVERTON, 2009; HACKNEY; EARHART, 2009; JEON et al., 2005; JEONG et al., 2005; NOREAU et al., 1995; PELC, 2002; SANDEL et al., 2005; SCHIMTT; FRÖLISH, 2007; SONG et al., 2004), nenhum ressaltou a influência da dançaterapia em pessoas com deficiência física por lesão nervosa cerebral. Para tal, instiga-se o desenvolvimento de mais estudos que ressaltem os efeitos de tal forma de terapia em longo prazo.

A presença de uma doença crônica está associada à piora da QV de uma população (HACKNEY; EARHART, 2009). A meta-análise realizada por Pang et al. (2006) mostrou que alguns autores demonstraram redução da QV de pacientes com lesão cerebral, quando comparados à população geral. Nesse trabalho, não foi possível essa comparação, devido à dificuldade em acessar os dados de QV do extrato da população brasileira que apresenta deficiência física analisada pelo SF-36.

Como visto, a presença de doença crônica estabelece a necessidade de um tratamento contínuo por um longo período, já que a idade avançada e a presença de comorbidades constituem fatores importantes na determinação da QV dessa população. A interação entre esses fatores, 
presentes em diferentes graus de intensidade em um dado paciente, pode explicar em parte a heterogeneidade dos resultados encontrados.

Entretanto, percebeu-se que a dançaterapia fomenta a interação de pessoas com deficiência como um todo, fato verificado mediante os dados relevantes acerca do estado geral da saúde, dos aspectos emocionais e sociais, que refletem na capacidade funcional dos participantes deste estudo.

\section{Considerações Finais}

Este estudo demonstrou redução da QV de pacientes com deficiência física e, ademais, que a dançaterapia pode promover significativa melhora de algumas dimensões da QV mediante o SF-36.

Os resultados obtidos sugerem apropriação ou adaptação do protocolo para avaliar a QV de pessoas nas diversas graduações de funcionalidade, para não excluir fatores essenciais em pessoas com alto grau de dependência para executar suas tarefas e, assim, não ser um fator limitante para se obter índices satisfatórios em todos os itens de QV, e não restringir os parâmetros que abrangem os aspectos funcionais, emocionais e sociais.

A dança, como terapia, interfere nos processos cognitivos para a elaboração do movimento por inquirir estímulos sensoriais que favorecem ampliação da capacidade funcional de pessoas com deficiência física.

Utilizar a dança como ferramenta para aprimorar o aparato neuromotor de pessoas com deficiência física mostrou-se importante nos aspectos emocionais, por embutir significado e motivação em fazer uma atividade ou participar de um programa de reabilitação que promove bem estar físico e emocional.

Em relação aos aspectos sociais, a dançaterapia viabiliza a inserção nos contextos sociais do indivíduo com limitações físicas por proporcionar a participação em atividades socioculturais.

Portanto, verificou-se que a dançaterapia possibilitou o aprimoramento da QV de indivíduos com deficiência física.

\section{Referências}

AKTAS, G.; OGCE, F. Dance as a therapy for cancer prevention. State Conservatory of Turkish Music, Department of Dance, Ege University, Izmir, Turkey. Asia Pacific Journal Cancer Prevention, v. 6, n. 3, p. 408-411, 2005.

ALPERT, P. T. et al. The effect of modified jazz dance on balance, cognition, and mood in older adults. Journal American Academic Nurse Practice, v. 21, n. 2, p. 108-115, 2009.

BUCHANAN, P.; ULRICH, B. D. The Feldenkrais Method: a dynamic approach to changing motor behavior. Research Quarterly for Exercise and Sport: American Alliance for Health, Physical Education, Recreation and Dance, v. 72, n. 4, p. 315-323, 2001.

CARR, J. H.; SHEPHERD, R. B. Enhancing physical activity and brain reorganization after stroke. Neurology Research International, v. 51, n. 5, p. 93-98, 2011.

CARR, J. H.; SHEPHERD, R. B. Should exercises and training be specific in motor learning? Journal of Physiotherapy, v. 58, n. 3, p. 206-207, 2012.

CAZE, C.; OLIVEIRA, A. Dança além da visão: possibilidades do corpo cego. Revista Pensar a Prática, v. 11, n. 3, p. 293-302, 2008. 
CICONELLI, R. M. et al. Tradução para a Língua Portuguesa e Validação do Questionário Genérico de Avaliação de Qualidade de Vida SF-36 (Brasil SF-36). Revista Brasileira de Reumatologia, v. 39, n. 3, p. 143-150, 1999.

COHEN, S. O.; WALCO, G. A. Dance/Movement therapy for children and adolescents with cancer. Cancer Practice, v. 7, n. 1, p. 34-42, 1999.

D'ALENCAR, B. P. et al. Biodance as process of existential renew for the elderly. Revista Brasileira de Enfermagem, v. 61, n. 5, p. 608-614, 2008.

DAPRATI, E.; SIRIGU, A.; NICO, D. Body and movement: consciousness in the parietal lobes. Neuropsychologia, v. 48, n. 3, p. 756-762, 2010.

DUIGNAN, D.; HEDLEY, L.; MILVERTON, R.: Exploring dance as a therapy for symptoms and social interaction in a dementia care unit. Nurse Times, v. 105, n. 30, p. 19-22, 2009.

EL-TAMAWY, M.S.; DARWISH, M.H.; KHALLAF, M. E. Effects of augmented proprioceptive cueson the parameters of gait of individuals with Parkinson`s disease. Annals of Indian Academy of Neurology, v. 15, n. 4, p. 267-272, 2012.

FORINASH, M. Music Therapy. Expressive Therapies. New York: Guilford Publishions, 2005.

FRONTERA, W. R.; SILVER, J. K. Essentials of physical medicine and rehabilitation. Lippincott Williams \& Wilkins Publishers, p. 512-529, 2002.

FUJITA, M. Feed-forward associative learning for volitional movement control. Neuroscience Research, v. 52, n. 2, p. 153-165, 2005.

HALL, S. A Identidade Cultural na Pós-modernidade. 6. ed. Rio de Janeiro: DP\&A, 2001.

HACKNEY, M. E.; EARHART, G. M.: Health-related quality of life and alternative forms of exercise in Parkinson disease. Parkinsonism Relat Disorders, v. 15, n. 9, p. 644-648, 2009.

HACKNEY, M. E. et al. Effects of tango on functional mobility in Parkinson's disease: a preliminary study. Journal Neurology Physical Therapy, v. 31, n. 4, p. 173-179, 2007.

JEON, M. Y. et al. The effects of a Korean traditional dance movement program in elderly women. Taehan Kanho Hakhoe Chi, v. 35, n. 7, p. 1268-1276, 2005.

JEONG, Y. J. et al. Dance movement therapy improves emotional responses and modulates neurohormones in adolescents with mild depression. International Journal Neuroscience, v. 115, n. 12, p. 1711-1720, 2005.

KANDEL, E. R. Perception of motion, depth and form. In: KANDEL, E.; SCHWARTZ, J. H.; JESSELL, T. M. Principles of Neuroscience. 3. ed. New York: Elsevier, 2001.

KAPLAN, R. M.: Quality of life, resource allocation, and the U.S. Health - care crisis. In: DIMSDALE, J. E.; BAUM, A. Quality of life in behavioral medicine research. New Jersey: Lawrence Erlbau m Associates, 1995.

KATZ, H. Um, Dois, Três. A Dança é o pensamento do corpo. Belo Horizonte: FTD, 2005. 
KIM, S. J.; KWAK, E. E.; PARK, E. S.; CHO, S. R. Differential effects of rhythmic auditory stimulation and neurodevelopmental treatment/Bobath on gait patterns in adults with cerebral palsy: a randomized controlled trial. Clinical Rehabilitation, v. 26, n. 10, p. 904-914, 2012.

LABAN, R. A vision of dynamic space. London: Laban Archieves, compiled by Lisa Ullman, The Falmer Press, 1984.

LAUMER, U. et al. Therapeutic effects of the Feldenkrais method 'awareness through movement' in patients with eating disorders. Psychotherapy Psychosomatic Medicine Psychology, v. 47, n. 5, p. 170-180, 1997.

LLINÁS, El cérebro e el mito del yo. Colombia: Bogotá, 2002.

LOMAN, S. Dance/Movement Therapy. Expressive Therapies. New York: Guilford Publishions, 2005.

MEHLING, W. E. et al. Body awareness: a phenomenological inquiry into the common ground of mind-body therapies. Philosophy Ethics Humanities Medicine, v. 7, n. 6, p. 6, 2011.

MOHAPATRA, S.; ARUIN, A. S. Static and dynamic visual cues in feed-forward postural control. Experimental Brain Research, v. 224, n. 1, p. 25-34, 2013.

MOHAPATRA, S.; KRISHNAN, V.; ARUIN, A. S. Postural control in response to an external perturbation: effect of altered proprioceptive information. Experimental Brain Research, v. 217, n. 2, p. 197-208, 2012.

MORRIS, J.; PEREZ, D.; NOE, B. The use of quality of life data in clinical practice. Quality of Life Research, v. 7, p. 85-91, 1998.

NAONE, E. The dancer in nature. Thesis (S. M. in Science Writing). Massachusetts Institute of Technology, Department of Humanities, Graduate Program in Science Writing, 2007.

NOREAU, L. et al. Effects of a modified dance-based exercise on cardiorespiratory fitness, psychological state and health status of persons with rheumatoid arthritis. American Journal Physical Medicine Rehabilitation, v.74, n. 1, p. 19-27, 1995.

NYSTRÖM, K.; LAURITZEN, S. O. Expressive bodies: demented persons' communication in a dance therapy context. Health: An Interdisciplinary Journal for the Social Study of Health, Illness and Medicine, v. 9, n. 3, p. 297-317, 2005.

PALO-BENGTSSON, L.; WINBLAD, B.; EKMAN, S. L. Social dancing: a way to support intellectual, emotional and motor functions in persons with dementia. Journal Psychiatric Mental Health Nurse, v. 5, n. 6, p. 545-554, 1998.

PANG, M. Y. C. et al. The use of aerobic exercise training in improving aerobic capacity in individuals with stroke: a meta-analysis. Clinical Rehabilitation, v. 20, n. 2, p. 97-111, 2006.

PELC, Z. Therapeutic values of dance movement and its influence on psychomotor development of deaf persons as a form of socialization and integration with the environment. Wiad Lek, v. 55, n. 2, p. 845-849, 2002. 
PINHEIRO FILHO, F. A noção de representação em Durkheim. Lua Nova, v. 61, n. 8, p. 139-155, 2004.

RESENDE, C. O que pode um corpo? O método Angel Vianna de conscientização do movimento como um instrumento terapêutico. Physis: Revista de Saúde Coletiva, v. 18, n. 3, p. 563-574, 2008.

RIMMER, J. Physical Activity among adults with a disability. JAMA, v. 299, n. 11, 2008.

RÖSLER, A. et al. Skill learning in patients with moderate Alzheimer's disease: a prospective pilotstudy of waltz-lessons. International Journal Geriatric Psychiatry, v. 17, n. 12, p. 1155-1156, 2002.

ROUHIAINEN, L. Ways of knowing in dance and art. Council of Finland: The Finish Academy, 2007.

SACKS, O. The power of music. Brain, v. 129, n. 10, p. 2528-2532, 2006.

SANDEL, S. L. et al. Dance and movement program improves quality-of-life measures in breast cancer survivors. Cancer Nurse, v. 28, n. 4, p. 301-309, 2005.

SAPER, C. Brain stem modulation of sensation, movement, and consciousness. Encyclopedia of Human Brain. v. 3. Elsevier Science, 2002.

SEIXAS, L. C. Humanização da Assistência à Saúde. Boletim do Instituto de Saúde, n. 36, p. 4-6, 2005.

SCHMITT, B.; FRÖLICH, L. Creative therapy options for patients with dementia-a systematic review. Fortschr Neurology Psychiatric, v. 75, n. 12, p. 699-707, 2007.

SCHUTTINGA, J. A. Quality of life from a federal regulatory perspective. In: DIMSDALE, J. E.; BAUM, A. Quality of life in behavioral medicine research. New Jersey: Lawrence Erlbaum Associates, 1995.

SONG, R. et al. Comparisons of motivation, health behaviors, and functional status among elders in residential homes in Korea. Public Health Nurse, v. 21, n. 4, p. 361-371, 2004.

STEPHENS, J.; MILLER, T. Feldenkrais method in rehabilitation: using functional integration and awareness through movement to explore new possibilities. In: Complementary Therapies in Rehabilitation: Evidence for Efficacy in Therapy, Prevention, and Wellness. Philadelphia, 3. ed., Slack Incorporated, 2009.

STRAZZACAPPA, M. A educação e a fábrica de corpos: A dança na escola. Caderno CEDES, v. 21, n. 53, p. 69-83, 2001.

TAUBE, M. L. Estudo qualitativo do desenvolvimento da capacidade rítmica da criança. Ritmo Espontâneo e Ritmo Métrico. Revista Movimento, v. 5, n. 9, p. 4-16, 1998.

TEIXEIRA-MACHADO, L. A. A qualidade de vida de pessoas com deficiência pela dança. In: CHARLOT, B. Dança, Teatro e Educação na sociedade contemporânea. Ribeirão Preto: Alphabeto Editora, 2011. 
TSANG, H. W.; CHAN, E. P.; CHEUNG, W. M. Effects of mindful and non-mindful exercises on people with depression: a systematic review. British Journal Clinical Psychology, v. 47, n. 3, p. 303-322, 2008.

VELHO, G. Individualismo e Cultura: notas para uma antropologia da sociedade contemporânea. 8. ed. Rio de Janeiro: Jorge Zahar, 2008.

XIA, J.; GRANT, T. J. Dance therapy for people schizofrenia. Schizofrenia Bulletim, v. 35, n. 4, p. 675-676, 2009. 